\title{
Influence of Gd doping on the Structural and Optical Properties
} of $\mathrm{ZnO}$

\author{
Nurul Fadzilah Ab Rasid ${ }^{1,2}$, Siti Nooraya Mohd Tawil ${ }^{1, *}$, \\ Norhidayah Che Ani ${ }^{2}$ and Mohd Zainizan Sahdan ${ }^{2}$ \\ ${ }^{1}$ Department of Electrical and Electronics Engineering, Universiti Pertahanan Nasional Malaysia, \\ 57000 Sungai Besi, Kuala Lumpur, Malaysia \\ ${ }^{2}$ Microelectronics and Nanotechnology Shamsuddin Research Center (MiNT-SRC), Universiti Tun \\ Hussein Onn Malaysia, 86400 Parit Raja, Batu Pahat, Johor, Malaysia \\ *Corresponding author. Email: nooraya@upnm.edu.my
}

Keywords: zinc oxide, Gd-doping, spin-coating, structural properties, optical properties

\begin{abstract}
Doping transition metal or rare-earth metal ion are one of the most popular topics in semiconductors. In this work, gadolinium (Gd) doped zinc oxide thin films was prepared using the spin - coating technique with different concentrations in atomic percent (at. \%). The influences of rare-earth ions doped into the zinc oxide films were studied. The effects on the physical and optical properties of the films were investigated by field emission scanning electron microscope, x-ray diffraction, atomic force microscopy and ultraviolet-visible spectrophotometer. It was found that the properties of zinc oxide can be tuned by changing the concentration physical and optical of Gd.
\end{abstract}

\section{Introduction}

Zinc oxide $(\mathrm{ZnO})$ has recently attracted enormous attention among researchers due to their wide band gap energy of $3.37 \mathrm{eV}$ and higher exciton binding energy of $60 \mathrm{meV}$ at room temperature, which makes it a promising candidate for many applications such as electronic, optoelectronic, and information technology devices including sensors and solar cells [1-4]. Besides that, it has special properties such as long-term stability, low cost, environmental friendliness, excellent resistance radiation, and etc. Furthermore, $\mathrm{ZnO}$ is an excellent material when doped with a suitable dopant for a variety of photonic applications [5]. Doping is a popular technique for tuning the optical properties of $\mathrm{ZnO}$ system because they show a lot of absorption transitions in the visible region. Rare-earth, such as Gd may introduce extra functionality to the material system since a combination of magnetic and optical properties may be possible to fabricate combine functional devices on a single chip [6-7].

The thin films can be deposited by a number of different techniques such as sputtering, metal chemical vapor deposition (MOCVD), pulsed laser deposition (PLD) and sol-gel method [8]. Among this technique, sol-gel has advantages for its simplicity, low-cost, not required a vacuum, capable to produce large area of coating and good uniformity of thickness [9]. In this study, sol-gel spin-coating technique was used in the synthesis of $\mathrm{Gd}$ doped $\mathrm{ZnO}$ films on a glass substrate using different concentration. The effects of different concentrations were studied in details.

\section{Experimental Procedure}

\section{A. Solution Preparation}

Gd doped $\mathrm{ZnO}$ films were deposited onto a microscope glass substrate by sol-gel spin-coating techniques. As a starting material, zinc acetate dehydrate (ZAD), gadolinium acetate dehydrate, and 2-methoxyethanol and monoethanolamine (MEA) were used as a dopant, solvent and stabilizer, respectively. In this work, five different concentrations in atomic percentages which are $0 \%, 1 \%$, $2 \%, 4 \%$ and $8 \%$ were prepared. An aqueous solution containing same molarity of $0.4 \mathrm{~mol} \mathrm{ZAD}$ and MEA, followed by different Gd concentration for each solution was diluted in $30 \mathrm{ml}$ of 
2-methoxyethanol. The mixed solution was then vigorously stirred at $60{ }^{\circ} \mathrm{C}$ for $30 \mathrm{~min}$. Then, MEA was added drop by drop in this solution with constant stirring for $2 \mathrm{~h}$ until it becomes clear and homogeneous. All solutions prepared were aged at room temperature for $24 \mathrm{~h}$ before deposition process.

\section{B. Film Deposition}

After $24 \mathrm{~h}$, each of the solutions was spin-coated by putting a few drops onto the glass substrates. Subsequently, the samples were preheated at $250{ }^{\circ} \mathrm{C}$ on the hot plate for $3 \mathrm{~min}$ to evaporate the solvent. The process was repeated 10 times to obtain a thick film on an appropriate thickness. Lastly, all samples were annealed in the furnace at $500{ }^{\circ} \mathrm{C}$ for $1 \mathrm{~h}$ to remove organic residuals.

\section{Characterization Technique}

The surface topologies of the films were investigated using atomic force microscopy (AFM, Park System, XE-100). Meanwhile, the morphology of the films was measured using a field-emission scanning electron microscopy (FESEM, Joel, JSM-7600) and the crystanility of the films were characterized using an X-ray diffractometer (XRD, PANanalytical, xpert $^{3}$-pro). The films were optically characterized using an ultraviolet-visible spectrophotometer (UV-Vis, Shimadzu UV 1800).

\section{Result and Analysis}

\section{A. Morphological Analysis}

Fig. 1 represents the surface morphologies of the films. Generally, we can see that the size of the nanoparticles is homogeneous, but there is porous granular surface caused by different concentration $[2,10]$. As can be seen in Fig. 1 (a), the size of the nanoparticles forming virtually a sphere. When Gd concentration was increased, the size of the nanoparticles turned into smaller cluster grains and it is shown in Fig. 1 (b-e).

\section{B. Surface Topologies Analysis}

Fig. 2 shows the three-dimensional (3-D) surface topology images of the samples. A scanning probe microscope was used to observe the surface topologies of the films in a scan size of $5 \mu \mathrm{m} \times 5 \mu \mathrm{m}$ by non-contact mode. Based on Fig. 2 (a), (c) and (d), they have non-uniform grains and relatively rough surface. However, for Fig. 2 (b) and (e), we can see that the surface is quite smooth and more uniform grains. Therefore, it is concluded that different concentration has a substantial effect on the surface topology and the roughness of the samples. The root-mean-square average (RMS) roughness [11], as determined from the AFM measurement shows that the lowest surface roughness $(1.302 \mathrm{~nm})$ has been observed for $8 \%$ of Gd concentration and the highest surface roughness $(2.778$ $\mathrm{nm}$ ) for $0 \%$ of $\mathrm{Gd}$ concentration.

\section{Structural Analysis}

Fig. 3 shows the XRD spectra of $\mathrm{Gd}$ doped $\mathrm{ZnO}$ films deposited onto a glass substrate using different concentration. It was found that the $\mathrm{ZnO}$ growth is preferential of $\left(\begin{array}{lll}0 & 0 & 2\end{array}\right)$ orientation, whereas other peak such as $\left(\begin{array}{lll}1 & 0 & 0\end{array}\right),\left(\begin{array}{lll}1 & 0 & 1\end{array}\right)$ and $\left(\begin{array}{lll}1 & 0 & 3\end{array}\right)$ with comparatively lower intensity were also observed for all film. When Gd concentration was increased, the intensity of the XRD peak at $\left(\begin{array}{ll}0 & 0\end{array}\right.$ 2 ) was degraded. The results are in accordance with the FESEM findings where we can see that in XRD results, the existence of peak $\left(\begin{array}{lll}1 & 0 & 3\end{array}\right)$ at $0 \%, 1 \%$ and $2 \%$, is higher than $4 \%$ and $8 \%$. This also indicates, that the samples are non-uniform and relatively rough surface. The increasing of the crystallite size is indicated by the enhancement of the crystallinity and c-axis orientation of the films [12]. 


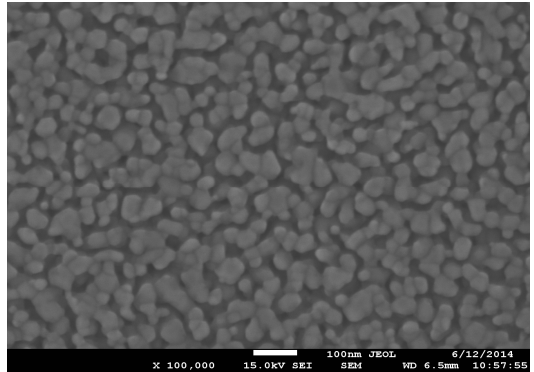

(a)

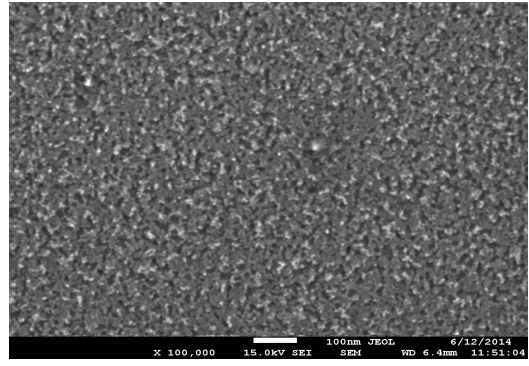

(b)

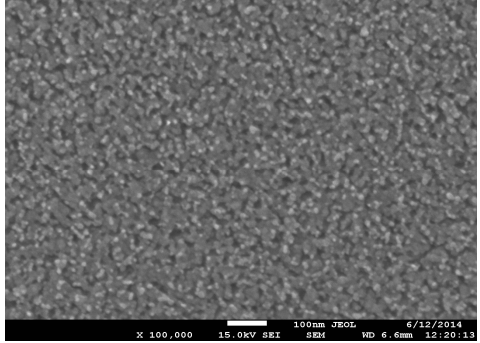

(c)

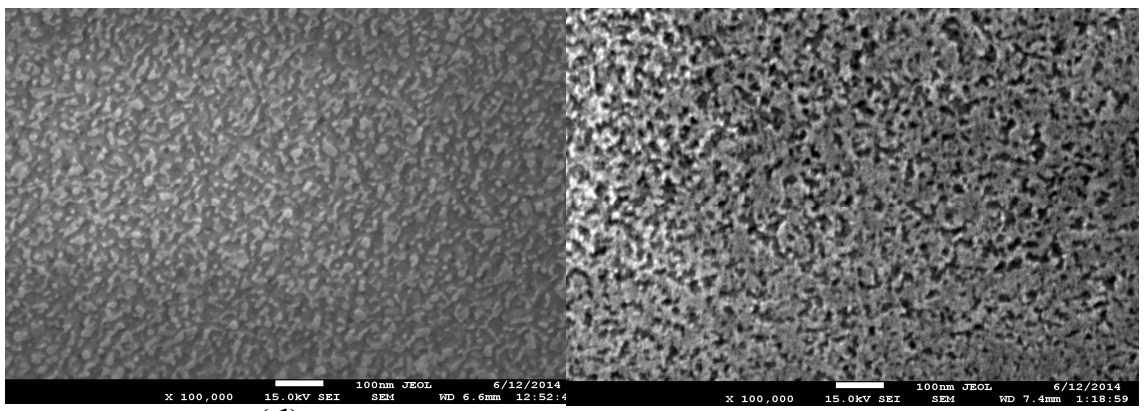

(d)

(e)

Fig. 1. FESEM image for $\mathrm{Gd}$ doped $\mathrm{ZnO}$ nanostructure at different concentration in atomic percentages (at. \%); (a) $0 \%$, (b) $1 \%$, (c) $2 \%$, (d) $4 \%$ and (e) $8 \%$, respectively

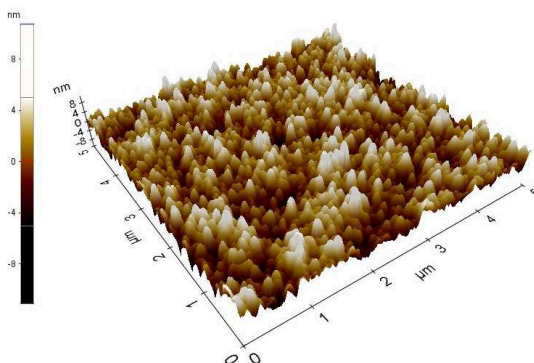

(a)

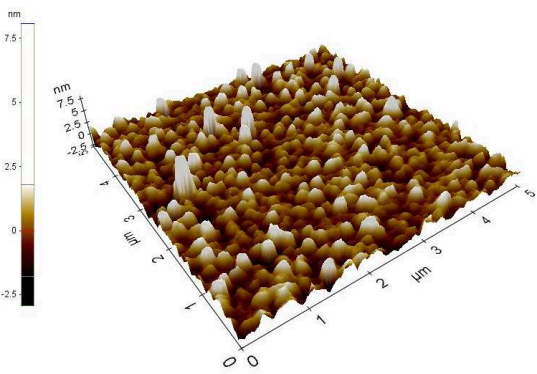

(d)

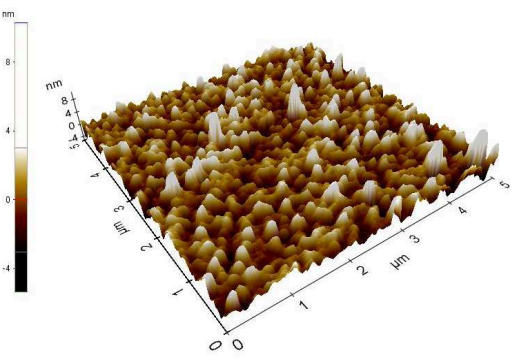

(b)

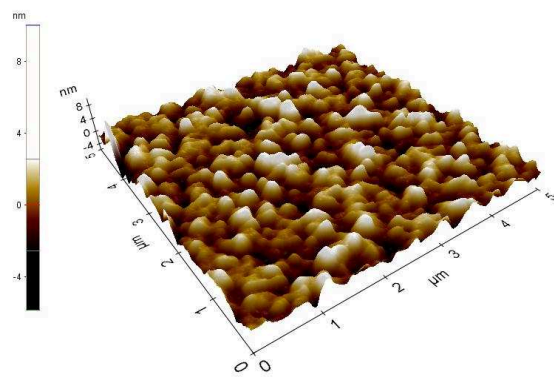

(e)

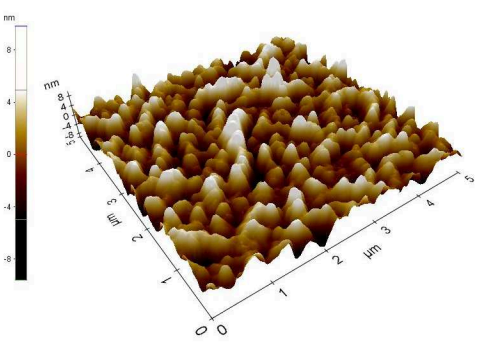

(c)

Fig. 2. AFM topologies image for $\mathrm{Gd}$ doped $\mathrm{ZnO}$ films at different concentration in atomic percentages (at. \%); (a) $0 \%$, (b) $1 \%$, (c) $2 \%$, (d) $4 \%$ and (e) $8 \%$, respectively 


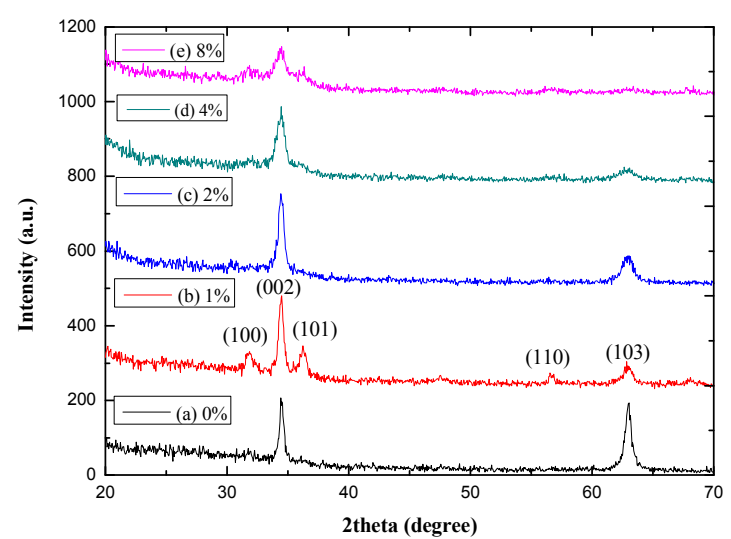

Fig. 3. XRD spectra of $\mathrm{Gd}$ doped $\mathrm{ZnO}$ films deposited using different Gd concentration; (a) $0 \%$, (b) $1 \%$, (c) $2 \%$, (d) $4 \%$ and (e) $8 \%$, respectively

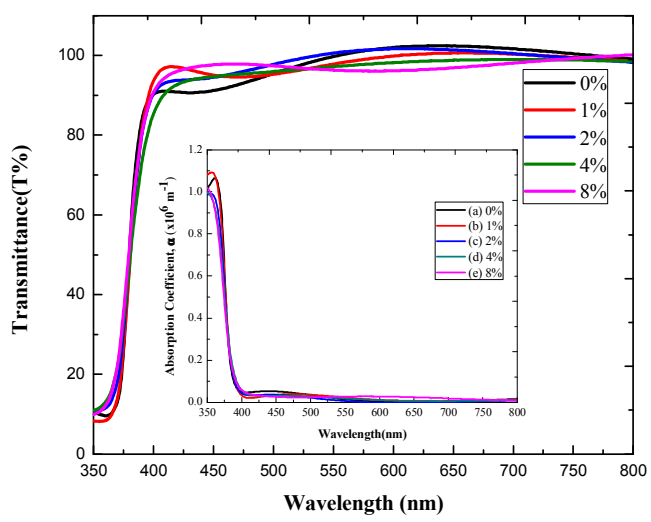

Fig. 4. Transmittance spectra of $\mathrm{Gd}$ doped $\mathrm{ZnO}$ films deposited using different $\mathrm{Gd}$ concentration. The intersect shows the absorption coefficient of $\mathrm{Gd}$ doped $\mathrm{ZnO}$; (a) $0 \%$, (b) $1 \%$, (c) $2 \%$, (d) $4 \%$ and (e) $8 \%$, respectively

\section{Optical Properties Analysis}

Fig. 4 presents the optical transmittance and intersect of absorption coefficient spectra of Gd doped $\mathrm{ZnO}$ films deposited using different concentrations measured at room temperature with wavelength ranging from $350-800 \mathrm{~nm}$. The transmission decreased sharply near the ultraviolet region at approximately $390 \mathrm{~nm}$ [1]. All films exhibit good transparency in the visible spectral range with an average transmission of approximately $94 \%$. Based on Fig. 4, we can see that for Gd concentration at $1 \%$ and $2 \%$, the signal is bouncing similarities with $\mathrm{ZnO}$ film, which indicates the film is highly crystalline whereas for $4 \%$ and $8 \%$, the films have a very nice slope which revealed the crystallinity is lower compared to the previous Gd concentration.

The absorption coefficient, $\alpha$, can be calculated based on the thickness of the thin films and transmittance data using Lambert's Law based on the relation (1):

$$
\alpha=\frac{1}{t} \ln \left(\frac{1}{T}\right)
$$

Where $t$ is the thickness of the thin film and $T$ is the transmittance of the thin film. The gradient of the absorption edge is an identification of the good crystallinity of the films. As reflected in the result, at $1 \%$, the film is highly crystalline because the signal is bouncing more than 1.0 of absorption coefficients compared to the other $\mathrm{Gd}$ concentration. Thus, the use of different $\mathrm{Gd}$ concentration affects the physical of the film, which causes the change in the optical properties.

\section{Conclusion}

In conclusion, Gd doped $\mathrm{ZnO}$ films have successfully deposited onto a glass substrate by sol-gel spin-coating techniques. Consequently, we have studied the effect of five different Gd concentration which is $0 \%, 1 \%, 2 \%, 4 \%$ and $8 \%$, respectively on the properties of Gd doped $\mathrm{ZnO}$ films. The FESEM studies show that when the Gd concentration was increased, the structures of the films are porous and the size of nanoparticles eventually became smaller cluster grains. Meanwhile, the XRD result shows that the intensity of the XRD peak at $\left(\begin{array}{lll}0 & 0 & 2\end{array}\right)$ was degraded when Gd concentration is changing. The AFM result revealed that Gd concentration which is $1 \%$ and $8 \%$ has a quite smooth with fine structure and uniform grains. However, for $0 \%, 2 \%$ and $4 \%$, they have non-uniform grains and relatively rough surface. Based on UV-this result, the films are highly crystalline when Gd 
concentration reached $1 \%$ and $2 \%$ similar to $\mathrm{ZnO}$ film, whereas $4 \%$ and $8 \%$ the crystallinity is lower. Generally, when transmittance is high, the absorption coefficient is lower. The results show that the use of different $\mathrm{Gd}$ concentration on the preparation of the solution affects the structure of the Gd doped $\mathrm{ZnO}$ film which causes a change in the properties of the films.

\section{Acknowledgement}

The authors would like to acknowledge the Ministry of Education for the financial support through FRGS grants vote 0823 from UTHM and FRGS/1/2013/SG06/UPNM/02/1 from UPNM.

\section{References}

[1] M. Malek, et al., "Sonicated sol-gel preparation of nanoparticulate $\mathrm{ZnO}$ thin films with various deposition speeds: The highly preferred c-axis (002) orientation enhances the final properties," Journal of Alloys and Compounds, vol. 582, pp. 12-21, 2014.

[2] M. Habibi and M. K. Sardashti, "Structure and morphology of nanostructured zinc oxide thin films Prepared by dip-vs. spin-coating methods," Journal of the Iranian Chemical Society, vol. 5, pp. 603-609, 2008.

[3] S. Ilican, et al., "Preparation and characterization of $\mathrm{ZnO}$ thin films deposited by sol-gel spin coating method," Journal of optoelectronics and advanced materials, vol. 10, pp. 2578-2583, 2008.

[4] X. Ma and Z. Wang, "The optical properties of rare earth Gd doped ZnO nanocrystals," Materials Science in Semiconductor Processing, vol. 15, pp. 227-231, 2012.

[5] H. Bahadur, et al., "Nano-structured ZnO films by sol-gel process," Indian Journal of Pure and Applied Physics, vol. 45, p. 395, 2007.

[6] S. Tawil, et al., "Studies on the $\mathrm{InGaGdN/GaN} \mathrm{magnetic} \mathrm{semiconductor} \mathrm{heterostructures}$ grown by plasma-assisted molecular-beam epitaxy," Journal of Crystal Growth, vol. 323, pp. 351-354, 2011.

[7] H. Ohno, et al., "(Ga, Mn) As: A new diluted magnetic semiconductor based on GaAs," Applied Physics Letters, vol. 69, pp. 363-365, 1996.

[8] M. Kamalasanan and S. Chandra, "Sol-gel synthesis of ZnO thin films," Thin Solid Films, vol. 288, pp. 112-115, 1996.

[9] M. Wang, et al., "Effect of preheating and annealing temperatures on quality characteristics of $\mathrm{ZnO}$ thin film prepared by sol-gel method," Materials Chemistry and Physics, vol. 97, pp. 219-225, 2006.

[10] J. Neamtu and M. Volmer, "The Influence of Doping with Transition Metal Ions on the Structure and Magnetic Properties of Zinc Oxide Thin Films," The Scientific World Journal, vol. 2014, 2014.

[11] F. Borghi, et al., "Nanoscale roughness and morphology affect the IsoElectric Point of titania surfaces," PLoS ONE, vol. 8, p. e68655, 2013.

[12] D. Bao, et al., "Sol-gel-derived $<\mathrm{i}>\mathrm{c}</ \mathrm{i}>$-axis oriented $\mathrm{ZnO}$ thin films," Thin Solid Films, vol. 312, pp. 37-39, 1998. 